\title{
Dual-modality optical biopsy of glioblastomas multiforme with diffuse reflectance and fluorescence: ex vivo retrieval of optical properties
}

\author{
Vinh Nguyen Du Le \\ John Provias \\ Naresh Murty \\ Michael S. Patterson \\ Zhaojun Nie \\ Joseph E. Hayward \\ Thomas J. Farrell \\ William McMillan \\ Wenbin Zhang \\ Qiyin Fang
}




\title{
Dual-modality optical biopsy of glioblastomas multiforme with diffuse reflectance and fluorescence: ex vivo retrieval of optical properties
}

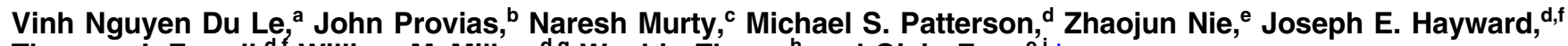 \\ Thomas J. Farrell, ${ }^{\mathrm{d}, \mathrm{f}}$ William McMillan, ${ }^{\mathrm{d}, \mathrm{g}}$ Wenbin Zhang, ${ }^{\mathrm{h}}$ and Qiyin Fang ${ }^{\mathrm{e}, \mathrm{i}, *}$ \\ ${ }^{a}$ McMaster University, Radiation Sciences Graduate Program, Hamilton, Ontario, Canada \\ ${ }^{b}$ McMaster University, Department of Anatomical Pathology, Hamilton, Ontario, Canada \\ 'McMaster University, Department of Surgery, Hamilton, Ontario, Canada \\ duravinski Cancer Centre, Hamilton, Ontario, Canada \\ eMcMaster University, School of Biomedical Engineering, Hamilton, Ontario, Canada \\ ${ }^{f}$ McMaster University, School of Interdisciplinary Science, Hamilton, Ontario, Canada \\ 9McMaster University, Department of Oncology, Hamilton, Ontario, Canada \\ hShanghai Jiaotong University Medical School, Shanghai 9th People's Hospital, Shanghai, China \\ 'McMaster University, Department of Engineering Physics, Hamilton, Ontario, Canada
}

\begin{abstract}
Glioma itself accounts for $80 \%$ of all malignant primary brain tumors, and glioblastoma multiforme (GBM) accounts for $55 \%$ of such tumors. Diffuse reflectance and fluorescence spectroscopy have the potential to discriminate healthy tissues from abnormal tissues and therefore are promising noninvasive methods for improving the accuracy of brain tissue resection. Optical properties were retrieved using an experimentally evaluated inverse solution. On average, the scattering coefficient is 2.4 times higher in GBM than in low grade glioma (LGG), and the absorption coefficient is $48 \%$ higher. In addition, the ratio of fluorescence to diffuse reflectance at the emission peak of $460 \mathrm{~nm}$ is 2.6 times higher for LGG while reflectance at $650 \mathrm{~nm}$ is 2.7 times higher for GBM. The results reported also show that the combination of diffuse reflectance and fluorescence spectroscopy could achieve sensitivity of $100 \%$ and specificity of $90 \%$ in discriminating GBM from LGG during ex vivo measurements of 22 sites from seven glioma specimens. Therefore, the current technique might be a promising tool for aiding neurosurgeons in determining the extent of surgical resection of glioma and, thus, improving intraoperative tumor identification for guiding surgical intervention. $\odot 2017$ Society of Photo-Optical Instrumentation Engineers (SPIE) [DOI: 10.1117/1.JBO.22.2.027002]
\end{abstract}

Keywords: optical properties; diffuse reflectance spectroscopy; fluorescence spectroscopy; glioblastoma multiforme; low grade glioma.

Paper 160474RR received Jul. 7, 2016; accepted for publication Jan. 12, 2017; published online Feb. 3, 2017.

\section{Introduction}

Glioma is a term used to describe all tumors arising from glial cells in the brain. There are three subgroups-astrocytoma, oligodendroglioma, and ependymoma-representing $30 \%$ of all primary brain tumors and $80 \%$ of all malignant primary brain tumors. ${ }^{1}$ Glioblastoma multiforme (GBM), the highest tumor grade in astrocytoma, accounts for nearly $55 \%$ of such tumors and affects over 12,000 people in the United States per year. ${ }^{1-4}$ GBM prognosis is very poor, and its median survival is reported to be from less than 14 months ${ }^{5}$ to 2 years. ${ }^{6,7}$ Currently, neurosurgeons define the extent of a brain tumor resection using techniques such as brain biopsy, ${ }^{8,9}$ intraoperative ultrasound, ${ }^{10}$ or visual inspection with the assistance of preoperative magnetic resonance (MR) images. ${ }^{11}$ Unfortunately, each method has considerable limitations. Brain biopsy is invasive and, hence, limits the number of specimens taken, leading to large sampling errors. Brain tumors frequently resemble normal brain tissues in ultrasound images, causing incomplete removal of margins. ${ }^{12}$ In addition, the nonrigidity of brain tissues might cause positional shifts during the operation, making the defined margins on

*Address all correspondence to: Qiyin Fang, E-mail: qiyin.fang@mcmaster.ca preoperative MR images unreliable. ${ }^{13,14}$ This problem may lead to either unplanned resection of normal brain tissues or incomplete resection of a tumor. ${ }^{15,16}$ Therefore, a noninvasive method is desired to allow real-time identification of GBM tissue.

Early studies have shown that diffuse reflectance spectroscopy (DRS) has the potential for noninvasive detection of mucosal abnormalities via differences in tissue optical properties ${ }^{17-19}$ and that fluorescence spectroscopy has the ability to detect malignant tissues by analyzing fluorescence characteristics such as emission spectrum ${ }^{20,21}$ and decay lifetime. ${ }^{22,23}$ Optical properties including absorption coefficient $\left(\mu_{\mathrm{a}}\right)$ and reduced scattering coefficient $\left(\mu_{\mathrm{s}}^{\prime}\right)$ are wavelength-dependent quantities that govern light propagation in tissues. ${ }^{24-27}$ In human mucosa, the principal absorbers are hemoglobin in blood, collagen crosslinks, nicotinamide adenine dinucleotide (NADH), and flavin adenine dinucleotide (FAD). ${ }^{28-30}$ Meanwhile, density and the morphology of cells are the main determinants of the scattering signal. ${ }^{31}$ Tissue optical properties are tied to the concentration of absorbers and scatterers present in that tissue. For example, a high number density of cells leads to high $\mu_{\mathrm{s}}^{\prime 32}$ while high blood vessel density suggests high $\mu_{\mathrm{a}}{ }^{33,34}$ In Lin et al.'s studies, it was shown that 
DRS alone could be used to discriminate brain tumor from normal brain tissues during in vivo measurements of 12 patients with a sensitivity of $95 \%$ and a specificity of $66 \% .^{35}$

Although the emission peaks in the steady-state fluorescence spectrum could be used to identify the principal fluorophores, the fluorescence signal is usually distorted by absorption and scattering of absorbers and scatterers present in the tissue. ${ }^{36,37}$ To correct the measured fluorescence, and to enable tissue discrimination using steady-state fluorescence, diffuse reflectance (DR) must be used in parallel. ${ }^{36,37}$ By combining steady-state fluorescence and DR, Lin et al. were also able to identify infiltrating tumor margins from normal brain tissues with a sensitivity of $100 \%$ and a specificity of $76 \% .{ }^{38}$ In similar studies with the integrated system, Toms et al. could identify glioma margins of 24 glioma patients with a sensitivity of $94 \%$ and a specificity of $93 \%,{ }^{39}$ and Valdés et al. were able to achieve a sensitivity and specificity of $94 \%$ in differentiating two low grade gliomas (LGG) patients and five GBM patients. ${ }^{40}$ Without using DRS, Butte et al. showed that fluorescence spectroscopy alone had the potential to discriminate LGG with a sensitivity of $100 \%$ and a specificity of $98 \% .{ }^{22,23}$ Unfortunately, the technique suffered low sensitivity (47\%) for detection of high grade gliomas. ${ }^{22,23} \mathrm{In}$ these studies, however, the spectral analysis for specific tumor grades, especially for LGG and GBM, was not analyzed separately, ${ }^{35,38-40}$ and the optical properties were not recovered. 22,23,35,38,39 In general, intraoperative differentiation of GBM and LGG from normal tissues can help neurosurgeons determine the extent of neurosurgical resection and determine a treatment plan. ${ }^{40-42}$ Knowledge of optical properties can also be used to calculate oxygen saturation, which is expressed as the percentage of oxyhemoglobin in the total hemoglobin. ${ }^{43-45}$ Although Asgari et al. were able to show that oxygen saturation was lower for LGG groups $(36 \pm 21 \%)$ and higher for GBM $(52 \pm 18 \%)$ due to the existence of arteriovenous shunts and metabolic shunts in GBM, optical properties were not measured. ${ }^{46}$ Whereas attempts to extract optical properties of healthy brain tissues and gliomas were performed in vitro by Gebhart et al., ${ }^{47}$ and in vivo by Valdés et al., ${ }^{40}$ optical properties were averaged for all gliomas, and separated observations for LGG and GBM were not available. ${ }^{40,47}$

In this paper, ex vivo DR and fluorescence measurements were performed to differentiate GBM from LGG in a total of 22 sites of seven glioma patients. An experimental look-up table (LUT) combined with a fitting routine was used to extract optical properties from 350 to $700 \mathrm{~nm}$. The approach was validated on liquid tissue-simulating phantoms containing hemoglobin, polystyrene microspheres, and India ink.

\section{Materials and Methods}

\subsection{Optical Phantoms and the Inverse Solution}

Similar to a previous study, ${ }^{48}$ the inverse solution for extracting optical properties from the measured DR combines an experimental LUT and fitting algorithms. The LUT was generated by measuring the DR from tissue-simulating phantoms with known optical properties. To simulate tissue scattering, polystyrene microspheres with a diameter of $1 \mu \mathrm{m}$ (07310-15, Polysciences Inc., Pennsylvania) were used. To simulate tissue absorption, black India ink (Higgins Ink, Chartpak Inc., Massachusetts) was used. A $6 \times 4$ matrix of 24 phantoms consisting of six different concentrations of black India ink $(0.025 \%, 0.05 \%, 0.1 \%, 0.2 \%, 0.35 \%$, and $0.5 \%$ of the concentrated ink solution) and four different concentrations of microspheres $(0.2 \%, 0.45 \%, 0.7 \%$, and $1 \% \mathrm{w} / \mathrm{v})$ was created [Fig. 1(a)].

(a) Increase of absorption
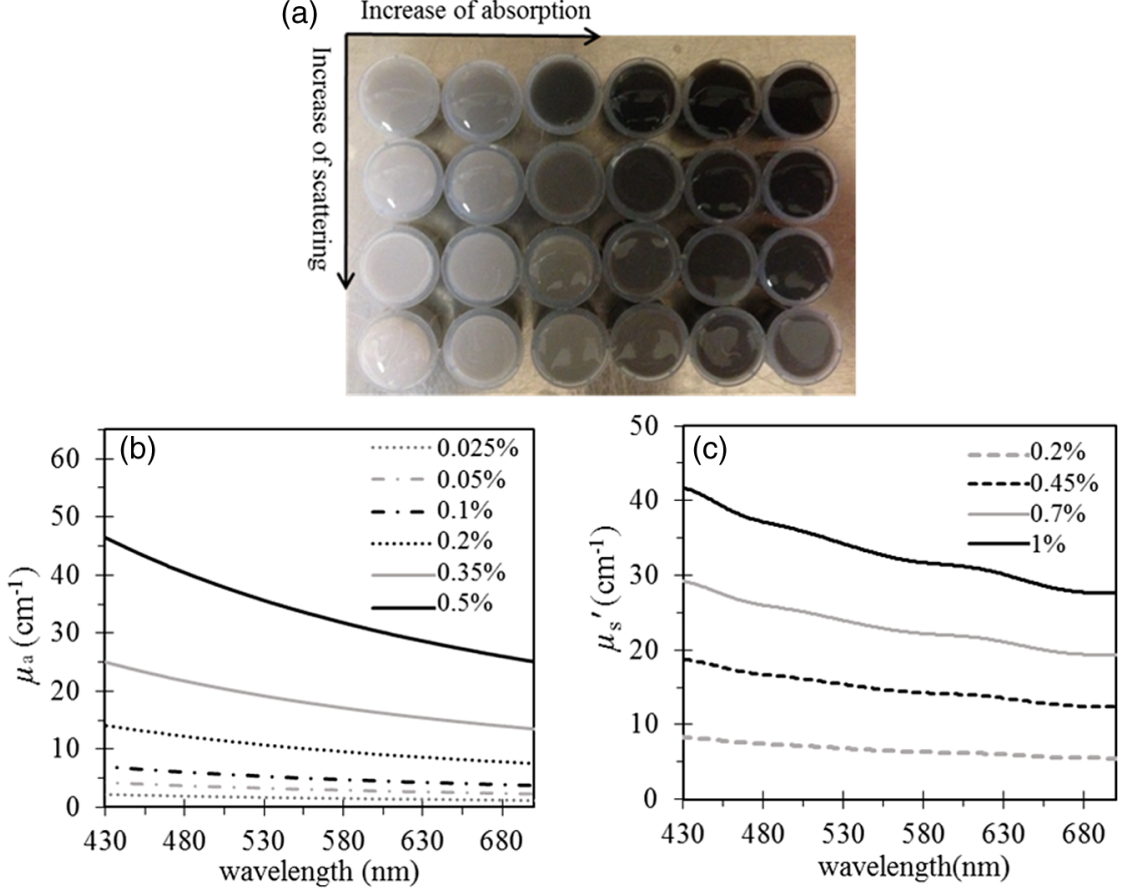

Fig. 1 Matrix of optical phantoms for LUT development: (a) top surface images of 24 phantoms captured with a standard digital camera, (b) absorption coeffients $\mu_{\mathrm{a}}$, and (c) reduced scattering coefficients $\mu_{\mathrm{s}}^{\prime}$. In (a), six concentrations of black India ink are $0.025 \%, 0.05 \%, 0.1 \%, 0.2 \%, 0.35 \%$, and $0.5 \%$, and four different concentrations of microspheres are $0.2 \%, 0.45 \%, 0.7 \%$, and $1 \% \mathrm{w} / \mathrm{v}$. These correspond to six spectra of $\mu_{\mathrm{a}}$ in (b) and four spectra of $\mu_{\mathrm{s}}^{\prime}$ in (c). 
Within the selected concentrations of India ink and microspheres, a $\mu_{\mathrm{a}}$ range of 0.05 to $47 \mathrm{~cm}^{-1}$ and a $\mu_{\mathrm{s}}^{\prime}$ range of 5 to $42 \mathrm{~cm}^{-1}$ were covered for a wavelength range 430 to $700 \mathrm{~nm}$ [Figs. 1(b) and 1(c)]. These optical properties were selected based on various reports studying human brain tissues and mucosal tissues..$^{20,29,30,47,49}$ To evaluate the LUT, selected phantoms containing ferrous stabilized hemoglobin (H0267, SigmaAldrich, Missouri) and microspheres with different concentrations were created. The DR from these phantoms was measured and the recovered optical properties were compared to target values.

In all phantoms, target optical properties were controlled and calculated by applying Beer-Lambert's law to the actual absorbance of pure solute absorbers (India ink or hemoglobin) measured with a spectrophotometer (Ultraspec 3000, Pharmacia Biotech Inc., New Jersey) for $\mu_{a}$, and by applying Mie theory to microsphere concentration for $\mu_{\mathrm{s}} .{ }^{50}$ Hemoglobin H0267 has an absorption spectrum similar to that of human blood with secondary absorption peaks at 540 and $580 \mathrm{~nm}$ and stable oxygen saturation. ${ }^{49,51,52}$ Polystyrene microspheres with a diameter of $1 \mu \mathrm{m}$ were preferred as scatterers because their scattering anisotropy is in a similar range to that of many biological tissues ( $g=0.89$ to 0.93 in UV-vis) and because their well-controlled size and index of refraction permits accurate calculation of scattering properties using Mie theory. ${ }^{45,50,53}$ Black India ink is widely used to simulate secondary absorbers in tissue due to its exponential decrease of absorption with wavelength, low cost, spectral stability, and low-fluorescence. ${ }^{54-57}$

To fit the optical properties, the least squares fitting routine fminsearch() in MATLAB ${ }^{\circledR}$ was used, so the absorption coefficients and the reduced scattering coefficients were constrained in the form of Eqs. (1) and (2). This optimization method is based on the Nelder-Mead simplex algorithm and has been used widely for spectral analysis in spectral imaging. ${ }^{58-60}$ The total absorption coefficient, $\mu_{\mathrm{a}}$, accounts for absorption of a primary absorber (human blood) and of secondary absorbers (i.e., NADH, FAD, and collagen). ${ }^{20,28,49}$ In general, the total absorption coefficients of all secondary absorbers can be described as an exponential decay with wavelength ${ }^{20,28}$ while the absorption coefficient of blood is determined predominantly by those of $\mathrm{Hb}$ and $\mathrm{HbO}_{2} \cdot{ }^{44,61} \mathrm{~A}$ similar fitting method for total absorption coefficients can be found elsewhere. ${ }^{48,61}$

$$
\begin{aligned}
\mu_{\mathrm{a}}(\lambda)= & A \cdot \mathrm{e}^{B} \cdot \lambda+\ln (10) \cdot f_{1} \\
& \cdot\left[f_{2} \cdot \varepsilon_{\mathrm{HbO} 2}(\lambda)+\left(1-f_{2}\right) \cdot \varepsilon_{\mathrm{Hb}}(\lambda)\right] .
\end{aligned}
$$

In Eq. (1), $A$ and $B$ are fitting coefficients that determine the contribution of secondary absorber so that $A\left(\mathrm{~cm}^{-1}\right)$ is the amplitude constant while $B\left(\mathrm{~nm}^{-1}\right)$ is the rate constant; $\lambda$ is the wavelength; $f_{1}(\mathrm{~mol} / \mathrm{l})$ is the total concentration of hemoglobin, $f_{2}$ (dimensionless) is the oxygen saturation, and $\varepsilon_{\mathrm{HbO} 2}\left(\mathrm{~cm}^{-1} \cdot \mathrm{M}^{-1}\right)$ and $\varepsilon_{\mathrm{Hb}}\left(\mathrm{cm}^{-1} \cdot \mathrm{M}^{-1}\right)$ are molar extinction coefficients of oxygenated and deoxygenated hemoglobin, respectively. In tissue measurements, $A, B, f_{1}$, and $f_{2}$ were calculated by applying the least squares fitting to the LUT-recovered $\mu_{\mathrm{a}}$ and the known spectra of $\varepsilon_{\mathrm{HbO} 2}$ and $\varepsilon_{\mathrm{Hb}} \cdot{ }^{62}$ In hemoglobin phantoms without collagen and $\mathrm{NADH}, A$ was set to zero and the extracted $f_{2}$ should be nearly $100 \%$ due to the nature of ferrous-stabilized hemoglobin. ${ }^{52}$ In human tissues, $\mu_{\mathrm{s}}^{\prime}$ is monotonically decreasing with wavelength, and the fitting equation for $\mu_{\mathrm{s}}^{\prime}$ can be expressed in the form of Eq. (2) where $a$ with unit of $\mathrm{cm}^{-1}$ is the factor characterizing magnitude of scattering, $b$ (dimensionless) is the factor that characterizes wavelength dependence of scattering, and $\lambda$ is the wavelength in nanometers $(\mathrm{nm}) .^{30,44,61}$ $\mu_{\mathrm{s}}^{\prime}(\lambda)=a \cdot \lambda^{b}$

\subsection{Brain Tissue Samples}

Fresh brain specimens were obtained from brain tissue removed during tumor resection surgery. The study protocol is approved by the McMaster/Hamilton Health Sciences Integrated Research Ethics Board, and patients consented to participate. Prior to the DRS measurement, each specimen was washed with saline, and the spectroscopic measurements were performed within $30 \mathrm{~min}$ of the surgery. A total of 22 sites were measured from specimens of seven patients. At each site, reflectance and fluorescence measurements were repeated four times to allow averaging and standard deviation calculation. Following the measurements, each site was marked with tissue marking dyes (Davidson Marking system, Bradley Products Inc., Minneapolis, Minnesota) in different colors. After the optical measurements, the specimens were preserved in formaldehyde and then cut into $5-\mu \mathrm{m}$-thick slices with hematoxylin and eosin stain. Tumor grade was assigned by a single pathologist (JP), using World Health Organization guidelines. ${ }^{63}$ The biopsy results identified four GBM patients (12 sites) and three LGG patients (10 sites). The surface area of tissue samples is at least five times larger than the surface area of the optical probe, which has a diameter of $3 \mathrm{~mm}$. Thickness of tissue samples is at least $0.5 \mathrm{~cm}$ and is much larger than the optical penetration depth range of 100 to $300 \mu \mathrm{m}$ for a human brain tumor at visible wavelengths. ${ }^{64}$

\subsection{Instruments}

DR signals between 430 and $700 \mathrm{~nm}$ were generated using a broadband light source (Dolan-Jenner MI-150, Edmund Optics, New Jersey), while fluorescence signals were generated using a solid-state laser (PNV-001525-140, Teem Photonics, Meylan, France) at $355 \mathrm{~nm}$ with 300 -ps pulses. Note that optical properties in the 350- to 430-nm range were extrapolated using the calculated parameters $\left(A, B, f_{1}, f_{2}, a\right.$, and $b$ from the fitting results). Measurements of both DR and steady state fluorescence (SSF) signals were performed with the same customized optical probe consisting of one source fiber and three detection fibers at source-detector collection distances (SDD) of 0.23, 0.59, and $1.67 \mathrm{~mm}$. A schematic and detailed description of the system can be found elsewhere. ${ }^{65-67}$ All fibers used in DRS and SSF measurements have a core diameter of $200 \mu \mathrm{m}$ and numerical aperture of 0.22 . After DRS and SSF measurements were performed, the fluorescence decay was recorded using a $400-\mu \mathrm{m}$ core optical fiber in the bundle. ${ }^{68}$ When observing highly absorbing phantoms, only background noise was collected with the furthest fibers, thus reducing the prediction accuracy of the inverse solution. Therefore, in the current study, only the two detection fibers closest to the source fiber were used to develop the LUT and to extract optical properties from the measured reflectance. To calculate the DR $R$ from the sample, the measured reflectance intensity of the sample was normalized to the reflectance intensity of a reflection standard with $99.9 \%$ reflectivity (Labsphere, Inc., New Hampshire) after subtracting background. $^{65}$

\section{Results}

\subsection{Validation of the Inverse Solution}

Figure 2(a) shows examples of DR spectra collected from six phantoms with the same microsphere concentration $(0.7 \% \mathrm{w} / \mathrm{v})$ 

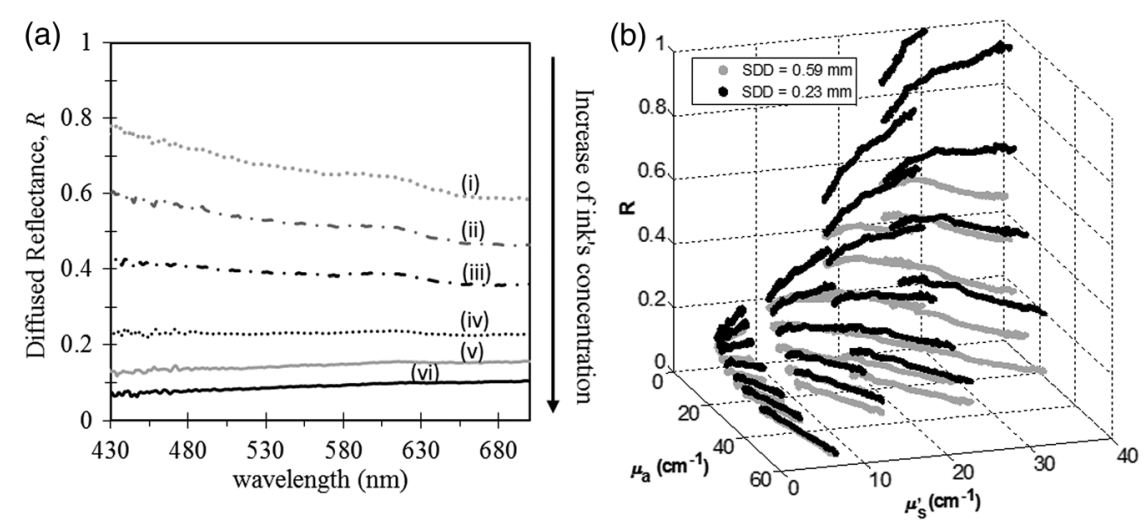

Fig. 2 (a) Examples of diffuse reflectance $R$ for six different ink concentrations while microsphere concentration remains constant and SDD $=0.23 \mathrm{~mm}$, and (b) $R$ as a sparse matrix mapped to optical property space $R\left[\mu_{\mathrm{a}}(\lambda), \mu_{\mathrm{s}}^{\prime}(\lambda)\right]$ for SDD $=0.23$ and $0.59 \mathrm{~mm}$. In (a), concentration of microsphere is $0.7 \%$ whereas concentrations of black India ink are $0.025 \%, 0.05 \%, 0.1 \%, 0.2 \%, 0.35 \%$, and $0.5 \%$, corresponding to six spectra (i) to (vi). In (b), the sparse matrix represents reflectance data per SDD collected from 24 phantoms (six ink concentrations $\times$ four microsphere concentrations).

and different India ink concentrations (from $0.05 \%$ to $1 \%$ ). Figure 2(b) shows the sparse matrix of DR collected from all 24 phantoms at SDD of 0.23 and $0.59 \mathrm{~mm}$. The LUT was evaluated with randomly selected phantoms consisting of hemoglobin and microspheres. Figures 3(a) and 3(b) compare the extracted and the target optical properties spectra for a selected phantom with microsphere concentration of $0.7 \% \mathrm{w} / \mathrm{v}$ and hemoglobin concentration of $8 \mathrm{mg} / \mathrm{ml}$. The target optical properties are those calculated with Beer-Lambert's law and Mie theory while the extracted values are those calculated from the inverse solution. As shown in Figs. 3(a) and 3(b), the method was able to retrieve $\mu_{\mathrm{a}}$ and $\mu_{\mathrm{s}}^{\prime}$ with average errors of $6 \%$ and $3 \%$, respectively, from 350 to $700 \mathrm{~nm}$. The intensive evaluation of the inverse solution was performed on a total of 10 hemoglobin
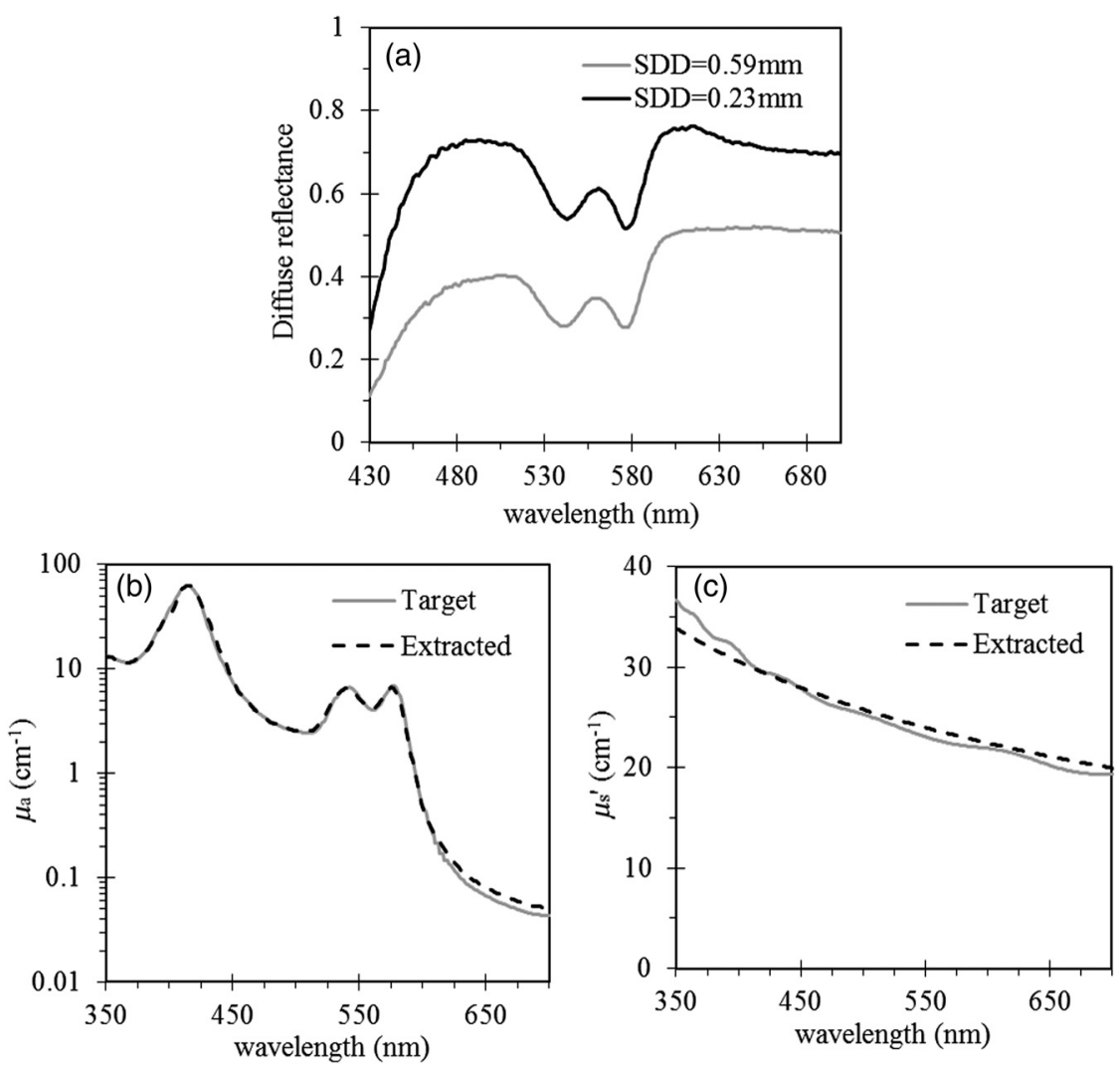

Fig. 3 An example of data analysis for a phantom with $\mathrm{Hb}$ concentration of $8 \mathrm{mg} / \mathrm{ml}$, microsphere concentration of $0.7 \%$ : (a) diffuse reflectance collected with fiber at SDD of 0.23 and $0.59 \mathrm{~mm},(\mathrm{~b}, \mathrm{c})$ theoretical (target) versus extracted optical properties. Equations (1) and (2) were used to extrapolate data in 350 to $430 \mathrm{~nm}$. In addition, $f_{1}$ and $f_{2}$ values of $7.9 \pm 0.8 \mathrm{mg} / \mathrm{ml}$ and $96 \% \pm 3 \%$ were obtained by using Eq. (1). 

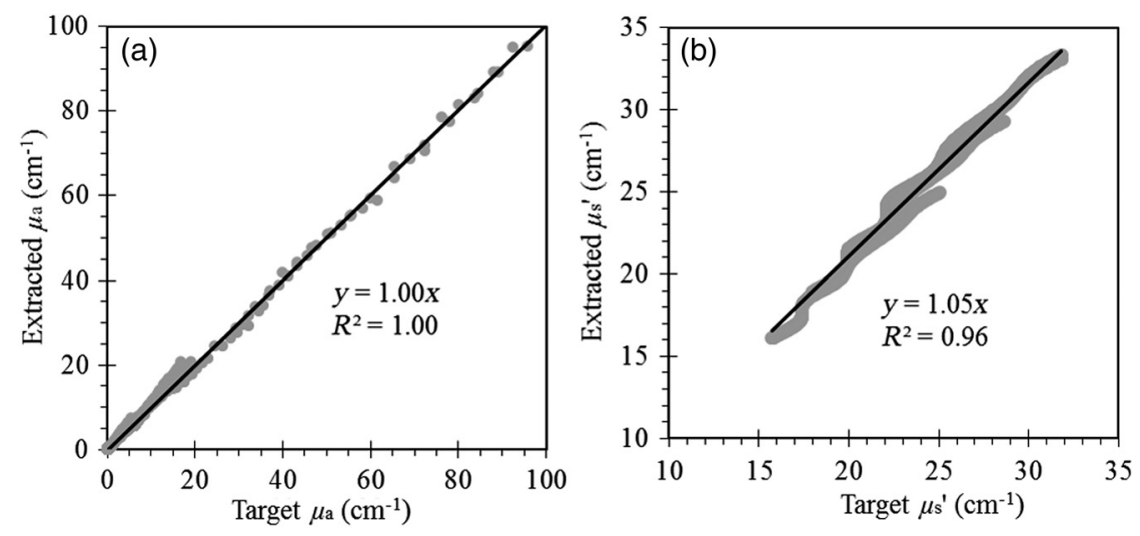

Fig. 4 Evaluation of LUT over 10 different hemoglobin phantoms. In general, average percentage errors of $9 \%$ and $6 \%$ were obtained for $\mu_{\mathrm{a}}$ and $\mu_{\mathrm{s}}^{\prime}$, respectively.

phantoms with a total of 3500 pairs of target optical properties in 430 to $700 \mathrm{~nm}$ (Fig. 4). On average, errors of $9 \%$ and $6 \%$ were observed for $\mu_{a}$ and $\mu_{\mathrm{s}}^{\prime}$, respectively. Furthermore, the fitting approach was able to retrieve $f_{1}$ and $f_{2}$ in all hemoglobin phantoms with average errors of $5.8 \%$ and $7 \%$, respectively. For example, the recovered $f_{1}$ and $f_{2}$ values for the phantom shown in Fig. 3 were $7.9 \pm 0.8 \mathrm{mg} / \mathrm{ml}$ and $96 \% \pm 3 \%$, respectively, versus target values of $8 \mathrm{mg} / \mathrm{ml}$ and $100 \%$, respectively.

\subsection{Brain Tissue Measurements}

Figure 5 compares the DR [Fig. 5(a)] and optical properties [Figs. 5(b) and 5(c)] measured for the GBM group and the LGG group. In Fig. 5, data were averaged over all 12 GBM sites and 10 LGG sites. On average over the entire spectrum (Fig. 5), DR was 3.2 times higher, $\mu_{\mathrm{a}}$ was $48 \%$ higher, and $\mu_{\mathrm{s}}^{\prime}$ was $140 \%$ higher for the GBM group. Data at $650 \mathrm{~nm}$ are also shown for comparison (Table 1). Note that $650 \mathrm{~nm}$ was selected because this is the region where blood absorption is small, and it is less likely that bleeding during surgery will affect tumor discrimination with optical measurement. ${ }^{35-39}$ The absorption coefficients were determined by the primary absorber (hemoglobin) and secondary absorbers. Below $600 \mathrm{~nm}$, absorption of hemoglobin dominated and determined the shape and intensity of the absorption spectrum. Hemoglobin absorption could be from blood within the tissue that has diagnostic value and/or blood on or close to the tissue surface that is the result of bleeding and has no diagnostic value. Although all
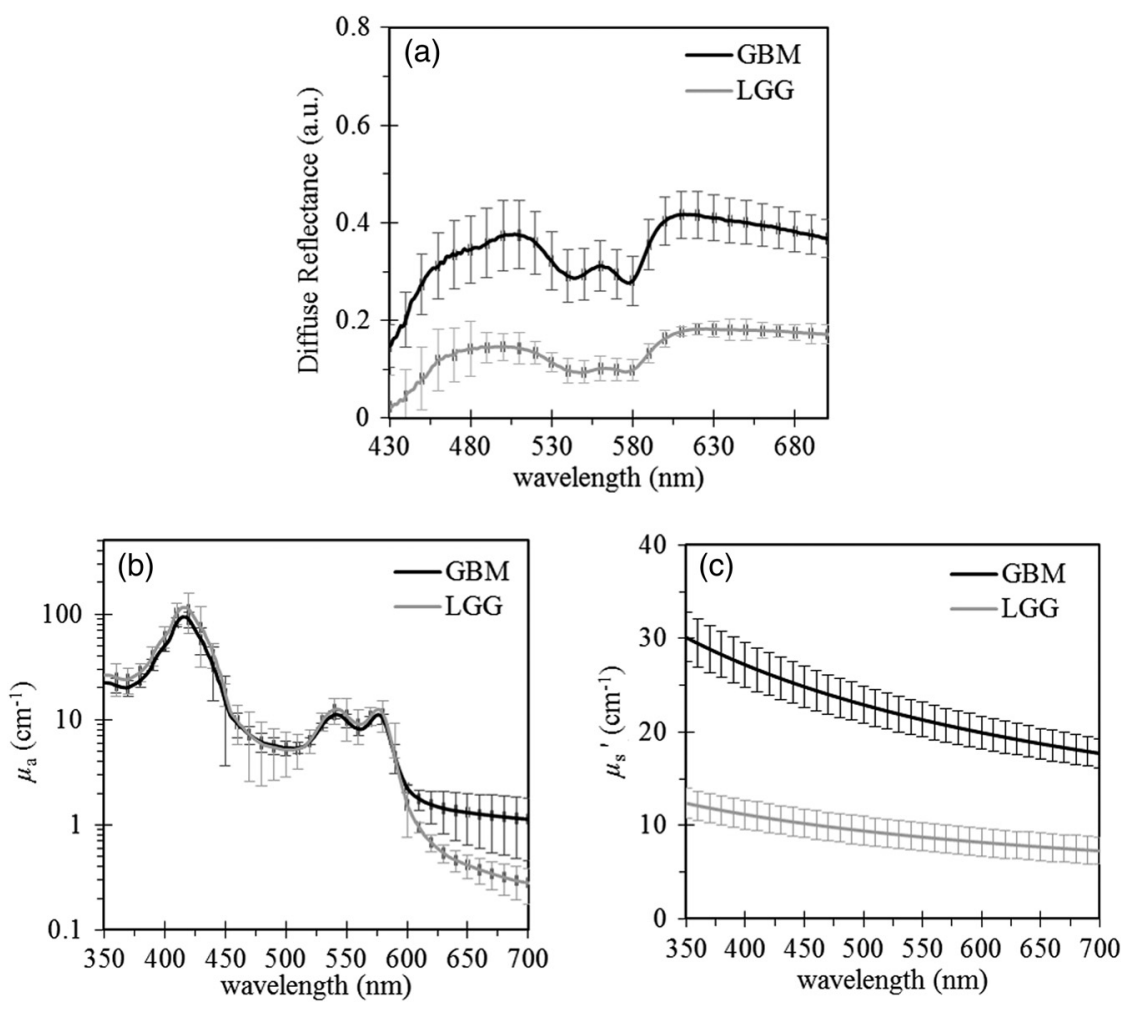

Fig. 5 LGG group versus GBM group average spectral analysis: (a) diffuse reflectance, (b) absorption coefficient $\mu_{\mathrm{a}}$, and (c) reduced scattering coefficient $\mu_{\mathrm{s}}^{\prime}$. Data were averaged over all sites (12 GBM sites and 10 LGG sites) and error bars are the standard deviation. 
Table 1 Average over all GBM and LGG sites: diffuse reflectance at $650 \mathrm{~nm}\left(R_{650}\right)$, optical properties at $650 \mathrm{~nm}\left(\mu_{\mathrm{a}, 650}\right.$ and $\left.\mu_{\mathrm{s}, 650}^{\prime}\right)$, ratio of fluorescence to reflectance at $460 \mathrm{~nm}(F / R)_{460}$, and fluorescence life-time at $460 \mathrm{~nm}\left(\tau_{460}\right)$.

\begin{tabular}{lll} 
Parameters & GBM & LGG \\
\hline$R_{650}$ (a.u.) & $0.44 \pm 0.03$ & $0.16 \pm 0.01$ \\
$\mu_{\mathrm{a}, 650}\left(\mathrm{~cm}^{-1}\right)$ & $1.31 \pm 0.11$ & $0.41 \pm 0.07$ \\
$\mu_{\mathrm{s}, 650}^{\prime}\left(\mathrm{cm}^{-1}\right)$ & $18.8 \pm 1.4$ & $7.7 \pm 0.7$ \\
$(F / R)_{460}$ (a.u.) & $9.9 \pm 1.2$ & $26.2 \pm 2.7$ \\
$\tau_{460}(\mathrm{~ns})$ & $3.2 \pm 0.3$ & $3.1 \pm 0.05$ \\
\hline
\end{tabular}

tissue samples were washed through with saline solution before measurement, a large portion of unwanted blood residues still remained and significantly affected the measured absorption coefficients below $600 \mathrm{~nm}$. As shown in Table 1, at $650 \mathrm{~nm}$ DR was 2.8 times higher, $\mu_{\mathrm{a}}$ was about 3 times higher, and $\mu_{\mathrm{s}}^{\prime}$ was 2.4 times higher for the GBM group. Figure 6 shows the DR [Fig. 6(a)] and optical properties [Figs. 6(b) and 6(c)] at $650 \mathrm{~nm}$ for all GBM and LGG sites. If we define sensitivity as the percentage of GBM sites that were correctly identified as GBM, and specificity as the percentage of LGG sites that were correctly identified as not GBM, the discrimination had a sensitivity of $100 \%(12 / 12)$ and specificity of $80 \%(8 / 10)$ if a cut-off at $20 \%$ was applied for DR at $650 \mathrm{~nm}$ to optimize the discrimination [Fig. 6(a)]. Sensitivity and specificity of $92 \%$ and $80 \%$ were achieved if a cut-off of $0.6 \mathrm{~cm}^{-1}$ was applied to $\mu_{\mathrm{a}}$ at $650 \mathrm{~nm}$ [Fig. 6(b)]. These numbers were $100 \%$ and $90 \%$ if a cut-off of $10 \mathrm{~cm}^{-1}$ was applied to $\mu_{\mathrm{s}}^{\prime}$ at $650 \mathrm{~nm}$ [Fig. 6(c)]. Although oxygen saturation was calculated at $83.4 \pm 17.3 \%$ for GBM and $55.4 \pm 9.9 \%$ for LGG using Eq. (1), the results might be affected by a long period of air exposure of the brain tissue specimen and were not used to optimize the discrimination for the ex vivo measurements.

Figure 7 shows the average fluorescence intensity with an emission peak at $460 \mathrm{~nm}$ [Fig. 7(a)], the average fluorescence lifetime [Fig. 7(b)], and the ratio of fluorescence and diffuse reflectance at $460 \mathrm{~nm}(F / R)_{460}$ versus diffuse reflectance at $650 \mathrm{~nm}\left(R_{650}\right)$ for GBM sites and LGG sites. Although the measured fluorescence signal could identify the characteristic emission peak of brain tissues at $460 \mathrm{~nm}$ [Fig. 7(a)], the measured fluorescence signal alone is not able to discriminate tumor types due to high tissue absorption in this wavelength range. To enable tumor discrimination, a graph of the ratio of fluorescence to diffuse reflectance at the emission peak $(F / R)_{460}$ versus $R_{650}$ was used instead. ${ }^{38,39}$ If a cut-off of 20 for $F_{460} / R_{460}$ was applied, sensitivity and specificity of $100 \%$ and of $90 \%$ were achieved. Although the measured fluorescence signal $\left(F_{460}\right)$ is distorted by absorption and $F_{460}$ alone cannot be used to differentiate various brain tumor types, $F_{460}$ can be corrected by using the measured reflection signal and the measured optical properties at the emission wavelength. ${ }^{37,65}$ In general, intrinsic fluorescence $\left(f_{460}\right)$ and fluorophore concentration are related to the ratio of $(F / R) .{ }^{37,65}$ As shown in Fig. 7(b) and Table 1, fluorescence lifetime alone was not able to discriminate GBM due to the high variation of life-time values, most likely caused by the low signal-to-noise ratio of the autofluorescence and high degree of heterogeneity in GBM. ${ }^{23}$ Figure 8 summarizes sensitivity and

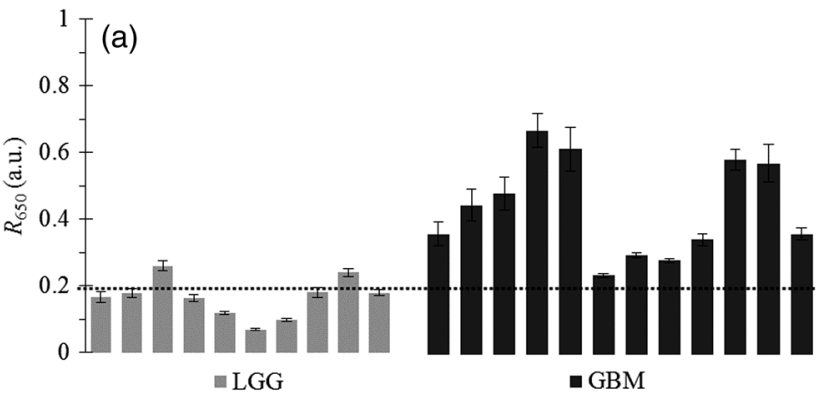

(b)
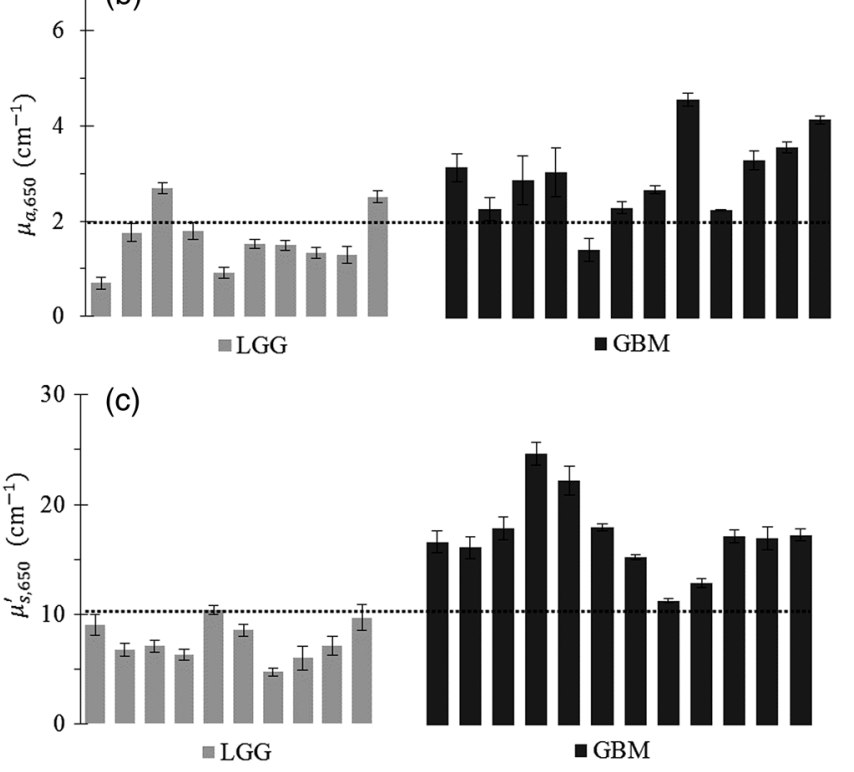

Fig. 6 LGG group versus GBM group: (a) diffuse reflectance at $650 \mathrm{~nm}\left(R_{650}\right)$, (b) $\mu_{\mathrm{a}}$ at $650 \mathrm{~nm}$, and (c) $\mu_{\mathrm{s}}^{\prime}$ at $650 \mathrm{~nm}$. Data at $650 \mathrm{~nm}$ were selected for due to small blood absorption in this region, and thus it is less likely for blood absorption to affect tumor discrimination. ${ }^{35-39}$

specificity when different parameters were used to discriminate GBM from LGG. In general, $R_{650}, \mu_{\mathrm{s}, 650}^{\prime}$ and ratio $(F / R)_{460}$ versus $R_{650}$ could achieve discrimination with a sensitivity of $100 \%$. Combining diffuse reflectance and steady-state fluorescence shows an increase in specificity from $80 \%$ to $90 \%$.

\section{Discussion and Conclusion}

Although the current LUT approach was similar to previous ones that aimed to study skin abnormalities, ${ }^{45,69,70}$ the current LUT covered optical properties of human brain tissues and mucosal tissues. More specifically, $\mu_{\mathrm{a}}$ range of 0.05 to $47 \mathrm{~cm}^{-1}$ and $\mu_{\mathrm{s}}^{\prime}$ range of 5 to $42 \mathrm{~cm}^{-1}$ were covered for a wavelength range 430 to $700 \mathrm{~nm}$. The developed inverse solution was thoroughly evaluated and was able to retrieve optical properties with an average relative error of $9 \%$ for $\mu_{\mathrm{a}}$ and $6 \%$ for $\mu_{\mathrm{s}}^{\prime}$ using phantoms with known optical properties. Furthermore, the developed LUT was used to establish broadband optical properties for GBM and LGG (Figs. 5 and 6). Within the errors of the inverse solution, ex vivo measurements showed that $\mu_{\mathrm{s}}^{\prime}$ for GBM was 2.4 times higher than that for LGG [Fig. 5(c)]. Because cell density in GBM is two to three times higher than that in $\mathrm{LGG}^{71,72}$ and the scattering probability is proportional to cellular density, ${ }^{32}$ the GBM group is expected to have higher $\mu_{\mathrm{s}}^{\prime}$ than the LGG group. Furthermore, it is believed that GBM is highly 

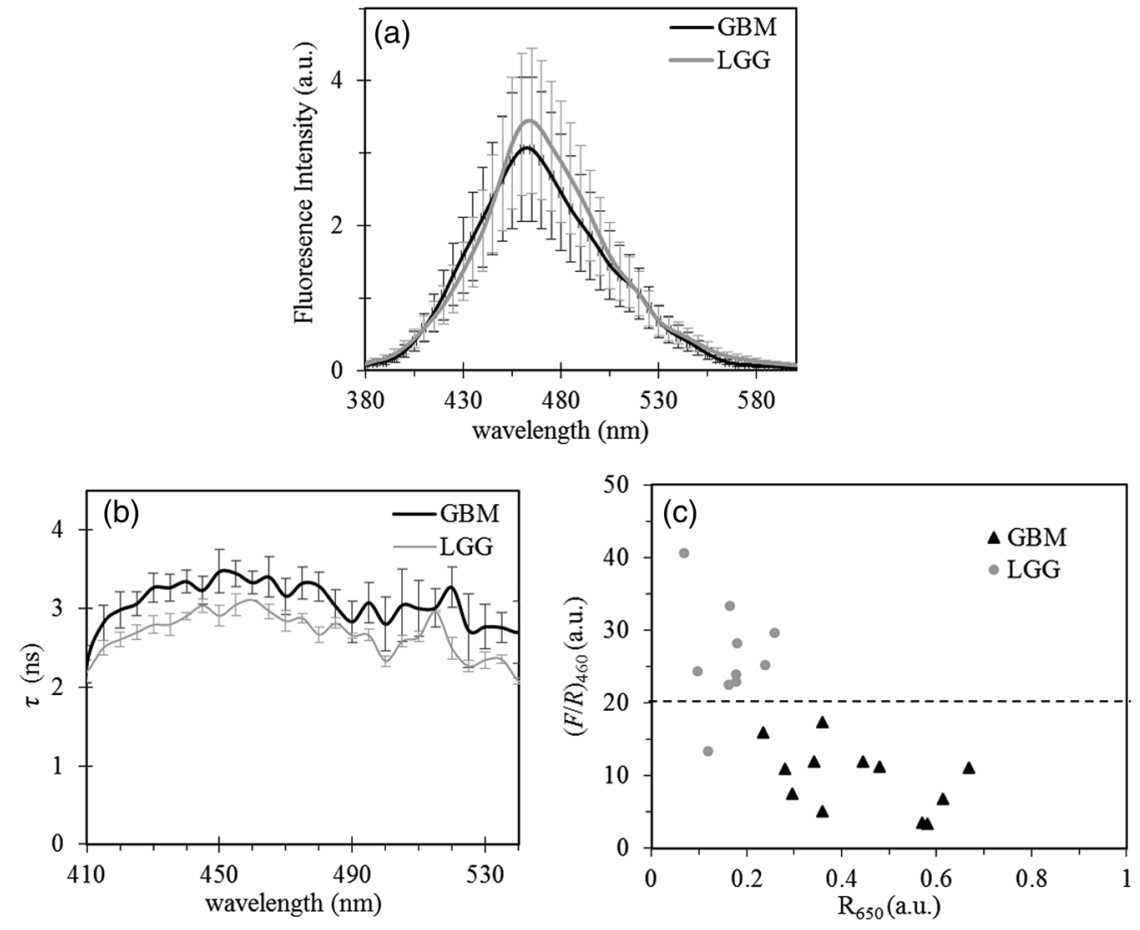

Fig. 7 LGG group versus GBM group: (a) SSF spectrum and (b) fluorescence life-time $\tau$ spectrum, (c) ratio of fluorescence to reflectance at $460 \mathrm{~nm}(F / R)_{460}$ versus reflectance at $650 \mathrm{~nm}\left(R_{650}\right)$. Fluorescence intensity has been normalized to integrating time and laser power corresponding to each measurement. In (a) and (b), data were averaged over all sites (12 GBM sites and 10 LGG sites) and error bars are the standard deviations.

vascular due to high degree of angiogenesis. ${ }^{73,74}$ This may imply that GBM has higher blood content or higher $\mu_{\mathrm{a}}$. In fact, with the observation at $650 \mathrm{~nm}$, the current study showed that $\mu_{\mathrm{a}}$ was about three times higher in GBM than that in LGG [Fig. 6(b) and Table 1]. Combining diffuse reflectance and steady-state fluorescence did not change the sensitivity (100\%), but increased the specificity from $80 \%$ to $90 \%$. Although optical measurements were performed during brain tumor resection, staging glioma (LGG versus GBM) based on their optical characteristics

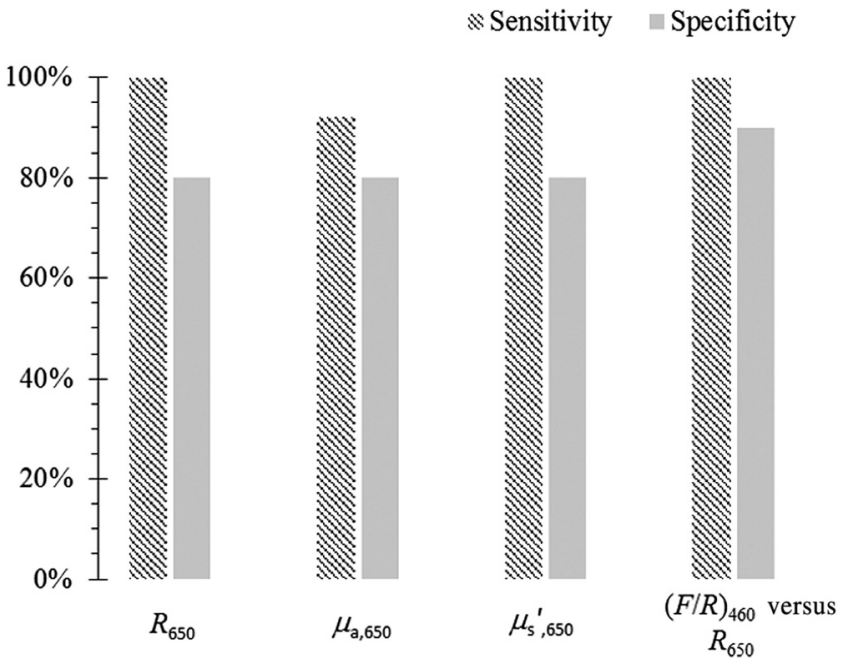

Fig. 8 Summary of sensitivity and specificity when using different parameters for GBM discrimination: diffuse reflectance at $650 \mathrm{~nm}$ $\left(R_{650}\right)$, optical properties at $650 \mathrm{~nm}\left(\mu_{\mathrm{a}, 650}\right.$ and $\left.\mu_{\mathrm{s}, 650}^{\prime}\right)$, and ratio $(F / R)_{460}$ versus $R_{650}$. has not been attempted. This study has established both optical properties and fluorescence properties for both stages. Similar to previous studies, ${ }^{35,38}$ the current study is limited by the small number of samples due to the limited number of glioma patients enrolled. Nevertheless, the current study has been able to discriminate GBM from LGG by accessing diffuse reflectance, optical properties measurement, and fluorescence (with diffuse reflectance). Although the measured oxygen saturation $\left(f_{2}\right)$ values were higher than that in Asgari et al.'s study, they showed a similar trend so that GBM has higher $f_{2}$ than LGG, specifically $83.4 \pm 17.3 \%$ for GBM and $55.4 \pm 9.9 \%$ for LGG. These numbers were $52 \pm 18 \%$ for GBM and $36 \pm 21 \%$ in the previous study. ${ }^{46}$ The difference in magnitude of $f_{2}$ between the two studies is mainly due to the fact that the current samples have been exposed to air during ex vivo measurements while intraoperative measurements were performed in the previous study. Meanwhile, it is believed that arteriovenous shunts and metabolic shunts can cause more oxygen than required in GBM and are responsible for high oxygen saturation levels in GBM. ${ }^{75}$ The preliminary results of the ex vivo measurements indicate that the current technique has the potential for in vivo discrimination of GBM by accessing the tumor's optical characteristics including diffuse reflectance, SSF, optical properties, and possibly oxygen saturation.

\section{Disclosures}

No conflicts of interest, financial or otherwise, are declared by the authors.

\section{Acknowledgments}

This project was supported in part by the Natural Sciences and Engineering Research Council (NSERC) of Canada, the Ontario 
Centers of Excellence, the Ontario Ministry of Research and Innovation, and the Canadian Cancer Society Research Institute (CCSRI). QF holds the Canada Research Chair in Biophotonics.

\section{References}

1. Q. T. Ostrom et al., "American brain tumor association adolescent and young adult primary brain and central nervous system tumors diagnosed in the United States in 2008-2012," Neuro Oncol. 18(1), i1-i50 (2015).

2. J. G. Scott et al., "Aggressive treatment is appropriate for glioblastoma multiforme patients 70 years old or older: a retrospective review of 206 cases," Neuro Oncol. 13(4), 428-436 (2011).

3. A. Agnihotri et al., "Glioblastoma, a brief review of history, molecular genetics, animal models and novel therapeutic strategies," Arch. Immunol. Ther. Exp. 61, 25-41 (2013).

4. K. R. Hess et al., "Adult glioma incidence trends in the United States, 1977-2000," Cancer 101, 2293-2299 (2004).

5. H. Golla et al., "Glioblastoma multiforme from diagnosis to death: a prospective, hospital-based, cohort, pilot feasibility study of patient reported symptoms and needs," Suppor. Care Cancer 22, 3341-3352 (2014).

6. M. E. Hegi et al., "MGMT gene silencing and benefit from temozolomide in glioblastoma," N. Engl. J. Med. 352, 997-1003 (2005).

7. R. Stupp et al., "Radiotherapy plus concomitant and adjuvant temozolomide for glioblastoma," N. Engl. J. Med. 352, 987-996 (2005).

8. N. Sanai et al., "An extent of resection threshold for newly diagnosed glioblastomas: clinical article," J. Neurosurg. 115, 3-8 (2011).

9. C. Scholtz, "Brain biopsy: the smear technique for neurosurgical biopsies," J. Roy. Soc. Med. 75, 61 (1982).

10. A. Alrawi, J. D. Trobe, M. Blaivas, and D. C. Musch, "Brain biopsy in primary angiitis of the central nervous system," Neurology 53, 858-858 (1999).

11. D. Ricard et al., "Primary brain tumours in adults," Lancet 379, 1984 1996 (2012).

12. O. Solheim et al., "Ultrasound-guided operations in unselected highgrade gliomas-overall results, impact of image quality and patient selection," Acta Neurochirurgica. 152, 1873-1886 (2010).

13. P. M. Matthews, M. Wylezinska, and T. Cadoux-Hudson, "Novel approaches to imaging brain tumors," Hematol. Oncol. Clin. North Am. 15, 609-630 (2001).

14. M. Lee et al., "The motor cortex shows adaptive functional changes to brain injury from multiple sclerosis," Ann. Neurol. 47, 606-613 (2000).

15. S. Sathornsumetee, J. N. Rich, and D. A. Reardon, "Diagnosis and treatment of high-grade astrocytoma," Neurol. Clin. 25, 1111-1139 (2007).

16. L. M. DeAngelis, "Brain tumors," N. Engl. J. Med. 344, 114-123 (2001).

17. G. Zonios et al., "Diffuse reflectance spectroscopy of human adenomatous colon polyps in vivo," Appl. Opt. 38, 6628-6637 (1999).

18. J. R. Mourant, "Spectroscopic diagnosis of bladder cancer with elastic light scattering," Lasers Surg. Med. 17, 350-357 (1995).

19. A. Amelink et al., "In vivo measurement of the local optical properties of tissue by use of differential path-length spectroscopy," Opt. Lett. 29, 1087-1089 (2004).

20. S. K. Chang et al., "Analytical model to describe fluorescence spectra of normal and preneoplastic epithelial tissue: comparison with Monte Carlo simulations and clinical measurements," J. Biomed. Opt. 9(3), 511-522 (2004).

21. S. K. Chang et al., "Model-based analysis of clinical fluorescence spectroscopy for in vivo detection of cervical intraepithelial dysplasia," $J$. Biomed. Opt. 11(2), 024008 (2006).

22. P. V. Butte et al., "Intraoperative delineation of primary brain tumors using time-resolved fluorescence spectroscopy," J. Biomed. Opt 15(2) 027008 (2010).

23. W. H. Yong et al., "Distinction of brain tissue, low grade and high grade glioma with time-resolved fluorescence spectroscopy," Front. Biosci. 11, 1255-1263 (2006).

24. T. J. Pfefer et al., "Reflectance-based determination of optical properties in highly attenuating tissue," J. Biomed. Opt. 8(2), 206-215 (2003).

25. A. J. Welch, M. J. C. van Gemert, and W. M. Star, "Definitions and overview of tissue optics," in Optical Thermal Response of Laser Irradiated Tissue, 2nd ed., A. J. Welch and M. J. C. van Gemert, Eds., Springer Science \& Business Media, New York (2011).
26. S. L Jacques and S. A. Prahl, "Optical Properties," 1998, http://omlc. org/education/ece532/class3/index.html (24 January 2017).

27. R. Graaff et al., "Similarity relations for anisotropic scattering in absorbing media," Opt. Eng. 32(2), 244-252 (1993).

28. R. Drezek et al., "Understanding the contributions of NADH and collagen to cervical tissue fluorescence spectra: modeling, measurements, and implications," J. Biomed. Opt. 6(4), 385-396 (2001).

29. S. L. Jacques, "Optical properties of biological tissues: a review," Phys. Med. Biol. 58, R37 (2013).

30. A. Kim and B. C. Wilson, "Measurement of ex vivo and in vivo tissue optical properties: methods and theories," in Optical Thermal Response of Laser Irradiated Tissue, A. J. Welch and M. J. C. van Gemert, 2nd ed., Springer Science \& Business Media, New York (2014)

31. R. Drezek, A. Dunn, and R. Richards-Kortum, "Light scattering from cells: finite-difference time-domain simulations and goniometric measurements," Appl. Opt. 38(16), 3651-3661 (1999).

32. J. R. Mourant et al., "Mechanisms of light scattering from biological cells relevant to noninvasive optical-tissue diagnostics," Appl. Opt. 37(16), 3586-3593 (1998).

33. A. Dellas et al., "Angiogenesis in cervical neoplasia: microvessel quantitation in precancerous lesions and invasive carcinomas with clinicopathological correlations," Gynecol. Oncol. 67, 27-33 (1997).

34. I. Pavlova et al., "Microanatomical and biochemical origins of normal and precancerous cervical autofluorescence using laser-scanning fluorescence confocal microscopy," Photochem. Photobiol. 77, 550-555 (2003).

35. W. C. Lin et al., "Diffuse reflectance spectroscopy for in vivo pediatric brain tumor detection," J. Biomed. Opt. 15(6), 061709 (2010).

36. Z. Volynskaya et al., "Diagnosing breast cancer using diffuse reflectance spectroscopy and intrinsic fluorescence spectroscopy," $J$. Biomed. Opt. 13(2), 024012 (2008).

37. M. G. Müller et al., "Intrinsic fluorescence spectroscopy in turbid media: disentangling effects of scattering and absorption," Appl. Opt. 40, 4633-4646 (2001).

38. W. C. Lin et al., "In vivo brain tumor demarcation using optical spectroscopy," Photochem. Photobiol. 73(4) 396-402 (2001).

39. S. A. Toms et al., "Intraoperative optical spectroscopy identifies infiltrating glioma margins with high sensitivity," Neurosurgery 57, 3823914 (2005).

40. P. A. Valdés et al., "Combined fluorescence and reflectance spectroscopy for in vivo quantification of cancer biomarkers in low-and high-grade glioma surgery," J. Biomed. Opt. 16(11), 116007 (2011).

41. W. Stummer et al., "Fluorescence-guided surgery with 5-aminolevulinic acid for resection of malignant glioma: a randomised controlled multicentre phase III trial," Lancet Oncol. 7(5), 392-401 (2006).

42. W. Stummer et al., "Extent of resection and survival in glioblastoma multiforme: identification of and adjustment for bias," Neurosurgery 62(3), 564-576 (2008).

43. T. O. McBride et al., "Spectroscopic diffuse optical tomography for the quantitative assessment of hemoglobin concentration and oxygen saturation in breast tissue," Appl. Opt. 38, 5480-5490 (1999).

44. R. Reif, O. A'Amar, and I. J. Bigio, "Analytical model of light reflectance for extraction of the optical properties in small volumes of turbid media," Appl. Opt. 46, 7317-7328 (2007).

45. N. Rajaram et al., "Design and validation of a clinical instrument for spectral diagnosis of cutaneous malignancy," Appl. Opt. 49, 142-152 (2010).

46. S. Asgari et al., "Intra-operative characterization of gliomas by nearinfrared spectroscopy: possible association with prognosis," Acta Neurochirurgica 145, 453-460 (2003).

47. S. C. Gebhart, W. C. Lin, and A. Mahadevan-Jansen, "In vitro determination of normal and neoplastic human brain tissue optical properties using inverse adding-doubling," Phys. Med. Biol. 51, 2011-2027 (2006).

48. N. Rajaram, T. H. Nguyen, and J. W. Tunnell, "Lookup table-based inverse model for determining optical properties of turbid media," $J$. Biomed. Opt. 13(5), 050501 (2008).

49. M. Friebel et al., "Determination of optical properties of human blood in the spectral range 250 to $1100 \mathrm{~nm}$ using Monte Carlo simulations with hematocrit-dependent effective scattering phase functions," J. Biomed. Opt. 11(3), 034021 (2006).

50. V. N. Du Le et al., "Measurements of extrinsic fluorescence in intralipid and polystyrene microspheres," Biomed. Opt. Express 5, 2726-2735 (2014). 
51. V. N. Du Le et al., "Vascular contrast in narrow band and white light imaging," Appl. Opt. 53, 4061-4071 (2014).

52. B. Yu et al., "Diffuse reflectance spectroscopy of epithelial tissue with a smart fiber-optic probe," Biomed. Opt. Express 5, 675-689 (2014).

53. Q. Liu, C. Zhu, and N. Ramanujam, "Experimental validation of Monte Carlo modeling of fluorescence in tissues in the UV-visible spectrum," J. Biomed. Opt. 8(2), 223-236 (2003).

54. B. W. Pogue and M. S. Patterson, "Review of tissue simulating phantoms for optical spectroscopy, imaging and dosimetry," J. Biomed. Opt. 11(4), 041102 (2006).

55. T. R. Wagner, W. G. Houf, and F. P. Incropera, "Radiative property measurements for India ink suspensions of varying concentration," Sol. Energy 25, 549-554 (1980).

56. H. Xu and M. Patterson, "Determination of the optical properties of tissue-simulating phantoms from interstitial frequency domain measurements of relative fluence and phase difference," Opt. Express 14, 64856501 (2006)

57. I. Barman et al., "Rapid and accurate determination of tissue optical properties using least-squares support vector machines," Biomed. Opt. Express 2, 592-599 (2011).

58. S. L. Jacques, R. Samatham, and N. Choudhury, "Rapid spectral analysis for spectral imaging," Biomed. Opt. Exp. 1(1) 157-164 (2010).

59. M. S. Twardowski et al., "Modeling the spectral shape of absorption by chromophoric dissolved organic matter," Mar. Chem. 89(1), 69-88 (2004).

60. X. Zhong, X. Wen, and D. Zhu, "Lookup-Table-based inverse model for human skin reflectance spectroscopy: two-layered Monte Carlo simulations and experiments," Opt. Express 22(2), 1852-1864 (2014).

61. P. R. Bargo et al., "In vivo determination of optical properties of normal and tumor tissue with white light reflectance and an empirical light transport model during endoscopy," J. Biomed. Opt. 10(3), 034018 (2005).

62. S. Prahl, "Tabulated molar extinction coefficient for hemoglobin in water," 1998, http://omlc.org/spectra/hemoglobin/summary.html (24 January 2017).

63. N. D. Louis et al., "The 2007 WHO classification of tumours of the central nervous system," Acta Neuropathol. 114(2), 97-109 (2007).

64. S. J. Madsen and B. C. Wilson, "Optical properties of brain tissue," in Optical Methods and Instrumentation in Brain Imaging and Therapy, A. J. Madsen Ed., Springer, New York (2013).

65. V. N. Du Le et al., "Experimental recovery of intrinsic fluorescence and fluorophore concentration in the presence of hemoglobin: spectral effect of scattering and absorption on fluorescence," J. Biomed. Opt. 20(12), 127003 (2015).

66. Z. Nie et al., "Integrated time-resolved fluorescence and diffuse reflectance spectroscopy instrument for intraoperative detection of brain tumor margin," IEEE J. Sel. Top. Quantum Electron. 22(3), 49-57 (2016).

67. Y. Yuan et al., "High throughput AOTF-based time-resolved fluorescence spectrometer for optical biopsy," Opt. Let. 34(7), 1132-1134 (2009).

68. D. J. Cappon et al., "A novel fibre optic probe design and optical property recovery algorithm for optical biopsy of brain tissue," J. Biomed. Opt. 18(10), 107004 (2013).

69. T. A. Erickson et al., "Lookup-table method for imaging optical properties with structured illumination beyond the diffusion theory regime," $J$. Biomed. Opt. 15(3), 036013 (2010).

70. B. S. Nichols, N. Rajaram, and J. W. Tunnell, "Performance of a lookup table-based approach for measuring tissue optical properties with diffuse optical spectroscopy," J. Biomed. Opt. 17(5), 057001 (2012).

71. K. M. Gauvain et al., "Evaluating pediatric brain tumor cellularity with diffusion-tensor imaging," Am. J. Roentgenol. 177(2), 449-454 (2001).
72. D. J. Brat et al., "Pseudopalisades in glioblastoma are hypoxic, express extracellular matrix proteases, and are formed by an actively migrating cell population," Cancer Res. 64(3), 920-927 (2004).

73. P. P. Pramanik et al., "Hypercellularity components of glioblastoma identified by high b-value diffusion-weighted imaging," Int. J. Rad. Oncol. Biol. Phys. 92, 811-819 (2015).

74. S. Das and P. A. Marsden, "Angiogenesis in glioblastoma," N. Engl. J. Med. 369, 1561-1563 (2013)

75. J. G. Ojemann et al., "Increased functional vascular response in the region of a glioma," J. Cereb. Blood Flow Metab. 18, 148-153 (1998).

Vinh Nguyen Du Le received his PhD (2016) in medical physics and applied radiation sciences from McMaster University, his bachelor's (2009) and master's (2010) degrees in biomedical engineering from the Catholic University of America (Washington, DC). He was a research engineer in the Optical Diagnostics Laboratory at the Center for Devices and Radiological Health of Food and Drug Administration (Silver Spring, MD) from 2010 to 2012.

John Provias received his MD degree from McMaster University. He is currently an associate professor of pathology and molecular medicine, McMaster University. His is also the neuropathologist for Hamilton Regional Cancer Centre.

Naresh Murty received his BSc degree in biology from Dalhousie University and his MD degree from Kasturba Medical College (India). $\mathrm{He}$ is currently an associate professor in the Division of Neurosurgery, McMaster University. $\mathrm{He}$ is also the head of neurosurgery service at Hamilton General Hospital.

Michael S. Patterson recently retired as the director of medical physics at Juravinski Cancer Centre. He received his BSc degree from Queen's University, his MSc degree from McMaster University, and his PhD from the University of Toronto.

Zhaojun Nie received her $\mathrm{PhD}$ in biomedical engineering from McMaster University in 2014. She is currently a postdoctoral scientist at Cedars-Sinai Medical Center, Los Angeles.

Joseph E. Hayward is a medical physicist at the Juravinski Cancer Centre and an associate professor of the medical physics department at McMaster University. He received his PhD in lasers and electrooptics in engineering physics from McMaster University.

Thomas J. Farrell is a medical physicist at the Juravinski Cancer Centre and the chair of the medical physics department at McMaster University. He received his PhD from McMaster University.

William McMillan is an associate professor at McMaster University's Department of Oncology. He received his BSc and MSc degrees from McMaster University, a BA degree from Queen's University, and an MB.CHB degree from the University College.

Wenbin Zhang received a $\mathrm{PhD}$ in clinic stomatology, College of Stomatology, Shanghai Jiaotong University, and a master degree in clinic stomatology, XiangYa School of Medicine, Central South University.

Qiyin Fang is an associate professor of engineering physics and the chair of biophotonics at McMaster University. He received his BSc degree in physics from Nankai University, his MSc degree in applied physics, and a PhD in biomedical physics from East Carolina University. 